\title{
Análisis factorial confirmatorio de la Escala de Mandatos de Género*
}

Confirmatory Factorial Analysis of the Scale of Gender Mandates

\author{
Jaime Sebastián F. Galán ${ }^{a}$ \\ Universidad Autónoma de San Luis Potosí, México \\ ORCID: http://orcid.org/0000-0002-8801-5201 \\ Gerardo Macías Valadéz-Márquez \\ Universidad Autónoma de San Luis Potosí, Namibia \\ ORCID: http://orcid.org/0000-0001-7554-7383
}

a Autor de correspondencia. Correo electrónico: sebastian.fgalan@uaslp.mx

Para citar este artículo: Galán, J. S. F., \& Valadéz-Márquez, G. M. (2019). Análisis factorial confirmatorio de la Escala de Mandatos de Género. Universitas Psychologica, 18(3). 1-9. https://doi.org/10.1114 4/Javeriana.upsy18-3.afce

\section{RESUMEN}

En México hay 30.6 millones de jóvenes de 15 a 29 años (25.7\% de la población total). El $49.4 \%$ de las mujeres no empleó anticonceptivo o condón en su primera relación sexual. Las conformaciones de género y las formas de socialización se transforman en los mandatos de género. Éstos, como categorías psicosociales, pueden ser entendidos como estereotipos prescriptivos que condicionan los procesos de socialización: nociones que determinan la masculinidad o feminidad desde una postura heteronormativa. Método: Se realizó un diseño no experimental analítico transeccional para obtener las propiedades psicométricas del instrumento mediante el análisis factorial confirmatorio. Con 446 jóvenes con una media de edad de 25.68 años (DE 16.8). Se aplicó la escala de mandatos de género a una muestra que presentara similar cantidad en hombres y mujeres. Se logró obtener una escala de cuatro factores, dos de mandatos clasificados como femeninos y dos masculinos. El instrumento logra un modelo factorial confirmatorio con los elementos psicométricos apropiados. Si bien uno de los factores aparece ligeramente bajo en confiabilidad, la escala resulta idónea para aproximaciones a las sexualidades heteronormativas.

Palabras clave

género; mandatos; escala; psicología social; psicometría.

\section{ABSTRACT}

In Mexico, there are 30.6 million young people aged 15 to 29 years $(25.7 \%$ of the total population). Of the women, $49.4 \%$ did not use contraceptives or condoms during their first sexual intercourse. Socialization and gender conformation are prone to evolve into gender mandates, which understood as psychosocial categories are prescriptive stereotypes that condition socialization processes: notions that determine masculinity or femininity from a heteronormative posture. Method: A nonexperimental, transectional analytical design was performed to obtain the psychometric properties of the instrument through confirmatory factor analysis. With 446 young people with a mean age of 25.68 years (DE 16.8). The scale of gender mandates was applied to a sample that presented a similar amount in men and women. A four-factor scale was obtained, having two mandates related to feminine gender mandates and two 
as masculine. The instrument achieves a confirmatory factorial model with the appropriate psychometric elements. Although one of the factors appears slightly low in reliability, the scale is ideal for approximations to heteronormative sexualities.

Keywords

gender; mandates; scale; psicología social; psicometría.

\section{Cautiverios y mandatos de género}

Para Lagarde (2005), la sexualidad constituye al individuo, es decir, lo obliga a la adscripción a grupos socioculturales genéricos, y va estableciendo condiciones a su forma de comportarse, así como a sus capacidades afectivas e intelectuales. La sexualidad se convierte en una identidad social, una de las primeras (Laqueur, 1990; Rudman \& Glick, 2008; Sanyal, 2012) que se va asociando con estereotipos. El heteropatriarcado, como sistema de organización sociocultural, propone y promueve modelos normativos acerca de lo que es ser un hombre masculino y una mujer femenina (Bosch, Ferrer, Ferreiro y Navarro, 2013), es decir, por medio éstos se van afianzando a las identidades sociales los estereotipos que condicionan la manera de comportarse.

Lagarde (2005) explora esta normatividad en las condiciones de los cautiverios de las mujeres. El cautiverio es una categoría antropológica que sintetiza el estado de la mujer en el mundo patriarcal, específicamente el de la privación de su libertad y la relación con el poder. Su base es la dependencia desigual, apuntalada por el dominio de los otros, ésta anula la "posibilidad de construir el deseo propio, el mundo personal, el Yo-misma" (p. 19). Un cautiverio es "un conjunto de límites materiales y subjetivos, de tabúes, prohibiciones, y obligaciones impuestas en la subordinación" (p. 641). La feminidad como cautiverio, se origina en los modos de vida y la cultura, se convierte en límites y obligaciones para la mujer que ella reproduce porque "no sabe ser de otra manera, no se atreve a serlo" (p. 20). Algunos cautiverios fundamentales son: la ideologización de la relación mujer-naturaleza (desde el nacimiento se concibe una forma de ser mujer), la centralidad del erotismo en la ideología de la pareja amorosa (vínculo entre sexo y amor) o su subsunción, y el cuerpo como eje de su sexualidad (cuerpo procreador para los otros).

Es a partir de la exploración de las condiciones de cautiverio de las mujeres que Lagarde (2005) hace referencia al concepto de mandatos de género, cautiverios no sólo entendidos en relación a la feminidad, sino también a la masculinidad. Los mandatos de género permiten explorar cómo los individuos o la cultura pueden normar el comportamiento de otros a partir de modelos que se imponen sobre el deber ser: mujer u hombre. Los cautiverios se estructuran en torno a la sexualidad (identidad social) y la relación con los otros (modos de vida, redes sociales y espacios culturales).

Los mandatos de género, como categoría psicosocial, se pueden entender como estereotipos prescriptivos que condicionan los procesos de socialización: tener una pareja o establecer relaciones sexuales, espacios en los cuales sería importante identificar y determinar su papel (Rudman \& Glick, 2008). Para Bosch et al. (2013). Hay vigencia en las expectativas, prescripciones (mandatos) y prohibiciones (cautiverios) que se hace a hombres y mujeres; se espera del varón que se comporte de acuerdo con las pautas masculinas, y que la mujer se comporte de acuerdo con las pautas femeninas. Los modelos normativos de masculinidad y feminidad actúan como mandatos de género, es decir, como marco de referencia socialmente compartido. Los mandatos culturales se transforman en mandatos de género (Yago, 2011), que condicionan el comportamiento principalmente en la sexualidad y la relación con los otros, no cumplirlos puede asociarse al miedo a ser rechazadas o no reconocidas por el entorno.

Para Lagarde (1996), ser hombre es ontológicamente: "ser-para-si-en-el mundo" (p. 62) y ser mujer "es ser-de-otros" (p. 61). De esta ontología se derivan los mandatos particulares de cada género, y que serán roles de obligado cumplimiento para hombres y mujeres. Briseño (2011) menciona tres mandatos de género femeninos: la maternidad, la abnegación femenina (ser buena y complaciente), y el 
mandato de la virginidad (no disfrutar de su propio cuerpo). En el caso de las mujeres, "no cumplir con estos mandatos se le sanciona con la amenaza de la pérdida del amor" (p. 93). Por su parte, Bosch et al. (2013) añaden que en la feminidad se exige a la mujer ser cuidadora, encontrarse predispuesta al amor, al rol de madre y su aspecto físico (la belleza como elemento para ser aceptada y valorada). La mujer es representada por características, como la dependencia emocional, la comprensión y el cuidado de los otros, que se pueden englobar en tres dimensiones: expresividad, sensibilidad y responsabilidad (Yago, 2011).

Para Lagarde (2005), la potencia erótica de los hombres "es uno de los fundamentos de la identidad" (p. 227). Así pues, el erotismo patriarcal es definido por su carácter de grandiosidad: "a mayor número de veces, a mayor duración (de la erección), a mayor número de coitos por sesión y, a mayor número de amantes, los hombres son considerados no sólo más potentes, sino más viriles, más machos, más hombres" (p. 227). La eyaculación es la síntesis del placer y del dominio del macho. Bosch et al. (2013) afirman que los mandatos masculinos incluyen no poseer ninguna de las características que se les suponen a las mujeres y sopesar éstas con sus opuestos. Éstos son: "ser racional, autosuficiente, controlador y proveedor, tener poder y éxito, ser audaz y resolutivo, ser seguro y confiado en sí mismo, no cuestionarse a sí mismo o las normas e ideales grupales" (p. 21).

Briseño (2011) menciona que hay tres mandatos masculinos básicos a) El mandato de la hombría: demostrar que no es mujer ni homosexual, es decir, "con la adquisición de la masculinidad hegemónica los hombres se ven obligados a suprimir emociones, debilidades, necesidades" (p. 96). b) El mandato de la virilidad: se asocia con un número alto de parejas, y para no ser tachados de carentes de virilidad, "no pueden negarse a tener relaciones sexuales por temor a que se ponga en tela de juicio su hombría" (p. 97). c) el mandato del hombre proveedor como aquel responsable de la alimentación y economía familiar.
Estos mandatos de género, aunque divididos en el binario masculino y femenino, como sugiere el feminismo de la diferencia (Carbó, 2017), atenúan las diferencias sexuales ya que se construyen en un contexto relacional, y no sólo cultural, es decir, su prescripción se asocia y se ejerce desde la relación que se establece con el otro.

Como se ha mencionado, la importancia de la identificación de los mandatos de género resulta una aportación psicosocial imprescindible, ya que, como estereotipos prescriptivos, orientan el comportamiento hacia condiciones de riesgo. Así lo reconocen los estudios que relacionan el género con factores como la violencia (MartínezMunguía \& Galán, 2015) y la sexualidad (Alfonso, 2010; Bombino, 2013), en la que conductas sexuales de riesgo y transmisión de enfermedades ponen en peligro la salud y bienestar de la persona. Y la identificación de los mismos posibilitaría la generación acciones para la prevención e intervención.

Instrumentos y medición de las variables de género en relación a la sexualidad

A continuación, se presenta una revisión de los instrumentos de medición recientes y algunos que se muestran con temáticas similares a la que proponemos.

La escala de asertividad sexual para mujeres (Sexual Assertiveness Scale [SAS] for Women) hecha por Morokoff et al. (1997), evalúa tres aspectos: iniciación (de la pauta sexual), rechazo (del acto sexual no deseado o inseguro) y prevención del embarazo e infecciones de transmisión sexual. Una de las limitantes que se podría atribuir a este instrumento, es que se enfoque en la población femenina y no dimensiona otros aspectos del género en conductas preventivas.

Otras escalas sobre género, que no tienen su traducción al español, se resumen en el compendio de escalas sobre género realizado por la United States Agency for International Development (USAID) y el C-Change project (2011) que contiene ocho escalas, de las cuales 
cinco relacionan género con aspectos de la sexualidad, (en la Tabla 1 se presenta la más relevante de ellas): Couple Communication on Sex Scale (predice el empoderamiento de la mujer para decidir cuándo tener o no sexo); Gender Beliefs Scale (predice comportamientos riesgosos para VIH, como es tener múltiples parejas sexuales); Gender Equipable Men (GEM) Scale (predice el uso del condón y contraceptivos, múltiples parejas sexuales y violencia de pareja); Gender Relation Scale (actitud frente a expectativas y rol de género, toma de decisiones acerca del sexo y la reproducción y la familia, violencia, comunicación); Sexual Relationship Power Scale (SRPS) (predice violencia física y sexual, violencia intima de pareja, uso de condón y fidelidad de pareja).

Yago y Tomás (2013) elaboraron y validaron un Cuestionario de anticoncepción y género, que evalúa la dimensión relacional en a) la actitud de la chica en sus aspectos de sumisa, ciega, dejarse llevar; y la actitud del chico en su aspecto de dominio; b) la identidad de género en su aspecto de maternidad en: eje central de la identidad y no idealizada; c) el rol de género femenino en sus aspectos: tradicional, inseguro, rol sexual y el cuidado (confianza, carencia básica y cuidado mutuo). Este cuestionario no está validado en población mexicana y sólo asocia el género en un ámbito específico de la sexualidad que es la anticoncepción, dejando fuera otros aspectos de prevención.

Sierra, Rojas, Ortega y Martín (2007) adaptaron la Double Standard Scale (DDS) en población española. Se habla de doble moral cuando se considera que los varones tienen más derechos para el coito prematrimonial que las mujeres. Esta doble moral sexual tradicional podría estar relacionada con la agresividad y/o violencia por parte del hombre en relaciones heterosexuales. La escala evalúa actitudes machistas y en esta aplicación española se les asoció con actitudes sexuales negativas (erotofobia y homofobia). Así, los individuos que puntuaron alto en erotofobia y homofobia se identificaron con una doble moral sexual, éstos también mostraron actitudes más negativas hacia el preservativo, (menos disposición a sugerir o usar condones durante sus relaciones sexuales). En general, los hombres mostraron más doble moral que las mujeres. Los autores consideran estos resultados predecibles, bajo la premisa de que el machismo fundamenta del mantenimiento del poder del hombre sobre la mujer. Aunque no se estableció una diferencia en la estructura factorial entre la aplicación a la población femenina y a la masculina (lo que significa que se pueda realizar aplicaciones conjuntas), la escala no evalúa aspectos de género en los que se ven implicadas las mujeres (pasividad, amor romántico, etc.), sino sólo con actitudes machistas que afectarían a mujeres de manera directa o indirecta.

La Tabla 1 presenta una comparativa de las características de los instrumentos para facilitar la construcción de la discusión y los hallazgos encontrados en temática de psicometría.

Tabla 1

Comparativo de instrumentos sobre género y sexualidad

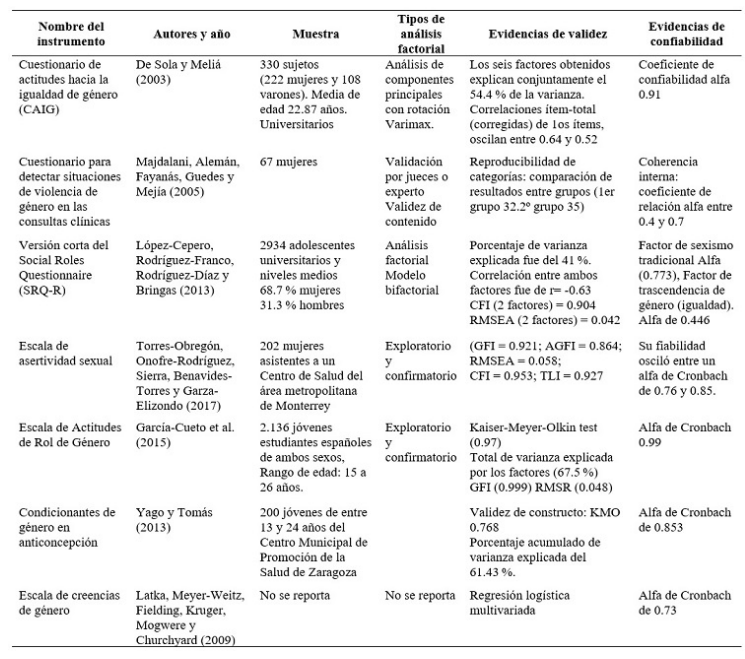

\section{Método}

Se realizó un diseño no experimental analítico transeccional para obtener las propiedades psicométricas del instrumento mediante el análisis factorial confirmatorio. 


\section{Muestra}

Se abordó a 446 jóvenes con una media de edad de 25.68 años (DE 16.8) 236 mujeres (52.9\%) y hombres 209 (46.9\%). Con niveles de estudio hasta primaria (33\%), secundaria (26\%), preparatoria $(17.9 \%)$ y universitarios $(23 \%)$ de San Luis Potosí y Aguascalientes, México. La muestra no fue aleatoria ya que se trabajó con participantes voluntarios.

\section{Instrumentos}

Escala de Mandatos de género para universitarios mexicanos (Macías-Valadez \& Luna-Lara, 2018). El análisis factorial exploratorio de la Escala de Mandatos de Género se aplicó a un total de 345 estudiantes [188 mujeres (54\%) y 157 hombres (46\%)] en distintas universidades públicas y privadas del municipio de San Luis Potosí, México, la cual pasó por una validación por expertos (de contenido). La escala resultó en un total de 29 ítems, que obtuvieron un Alfa de Cronbach de 0.899, con seis factores (cuyas alfas oscilan entre 0.755 y 0.862 ), cuyas dos dimensiones destacan aspectos relacionales en la relación sexual y de pareja. Además, se identifican el modelo de género al que pertenece, es decir, mandatos de género femeninos: pasividad (en la sexualidad), predisposición al amor, cuerpo (procuración de la belleza), abnegación. Y los masculinos: potencia erótica y virilidad.

\section{Procedimiento}

Se aplicó la escala de mandatos de género a una muestra que presentara similar cantidad en hombres y mujeres, que fuesen heterosexuales (por las posibles variaciones de los mandatos en sexualidades no heteronormativas). El análisis factorial confirmatorio se realizó con apoyo del programa IBM AMOS versión 21.0, con el método de rotación de máxima verosimilitud.

\section{Resultados}

Los resultados del análisis factorial confirmatorio se muestran en la Tabla 2, ahí se observa que el modelo inicial de la escala cuenta con los factores originales, mientras que el modelo definitivo se constituye solamente por cuatro factores. Se consideró conveniente renombrar los factores de predisposición al amor y el de abnegación por procreación y orientación rescatadora, respectivamente. Esto se debe a que al analizar la Figura 2, la cual presenta el modelo factorial confirmatorio, estas nuevas categorías representan mejor la intención estereotipada.

Tabla 2

Propiedades de los modelos factoriales confirmatorios

\begin{tabular}{lrc}
\hline Índices/puntuación & $\begin{array}{c}\text { Puntuación } \\
\text { Modelo inicial }\end{array}$ & $\begin{array}{c}\text { Modelo } \\
\text { final }\end{array}$ \\
\hline Cmin/df & 2.971 & 1.062 \\
$p$-valor del modelo & 0 & 0.375 \\
CFI & 0.883 & 0.999 \\
GFI & 0.845 & 0.987 \\
AGFI & 0.813 & 0.975 \\
SRMR & 0.0062 & 0.0011 \\
RMSEA & 0.067 & 0.012 \\
PCLOSE & 0 & 0.995 \\
TLI & 0.869 & 0.998 \\
\hline
\end{tabular}

Como se puede apreciar el modelo final (Tabla 3) tiene muchas mejores propiedades psicométricas, incluso, el modelo estadístico en los factores mantiene cierta congruencia con el modelo original, cuestión que se retomará en la discusión. Pero se puede interpretar que el instrumento cumple con todas las características propuestas para que el modelo confirmatorio sea adecuado. 
Tabla 3

Propiedades psicométricas de la escala en modelo inicial y final

\begin{tabular}{lrrrr}
\hline \multicolumn{1}{c}{ Factores } & $\begin{array}{c}\text { Confiabilidad } \\
\text { compuesta }\end{array}$ & AVE & MSV & $\begin{array}{c}\text { Máxima } \\
\text { confiabilidad } \\
\text { (H) }\end{array}$ \\
\hline Virilidad original & 0.823 & 0.609 & 0.531 & 0.833 \\
Potencia original & 0.802 & 0.585 & 0.271 & 0.925 \\
Abnegación original & 0.822 & 0.698 & 0.174 & 0.945 \\
Predisposición original & 0.684 & 0.522 & 0.171 & 0.951 \\
Cuerpo original & 0.728 & 0.573 & 0.159 & 0.957 \\
Pasividad original & 0.870 & 0.528 & 0.531 & 0.967 \\
Orientación rescatadora & & & & \\
último modelo & 0.820 & 0.696 & 0.134 & 0.821 \\
potencia último modelo & 0.802 & 0.585 & 0.168 & 0.923 \\
Procreación último modelo & 0.693 & 0.535 & 0.125 & 0.936 \\
Virilidad último modelo & 0.815 & 0.596 & 0.168 & 0.952 \\
\hline
\end{tabular}

En el segundo modelo continúa siendo ligeramente baja la confiabilidad del factor de predisposición al amor (posteriormente nombrado como procreación), sin embargo, se ha decidido conservarlo por su decidida aportación a la medición de los mandatos de género femeninos, además de que el resto de los elementos psicométricos, toman proporciones y puntajes adecuados.

\section{Figura 1}

Modelo inicial confirmatorio de la escala de mandatos de género

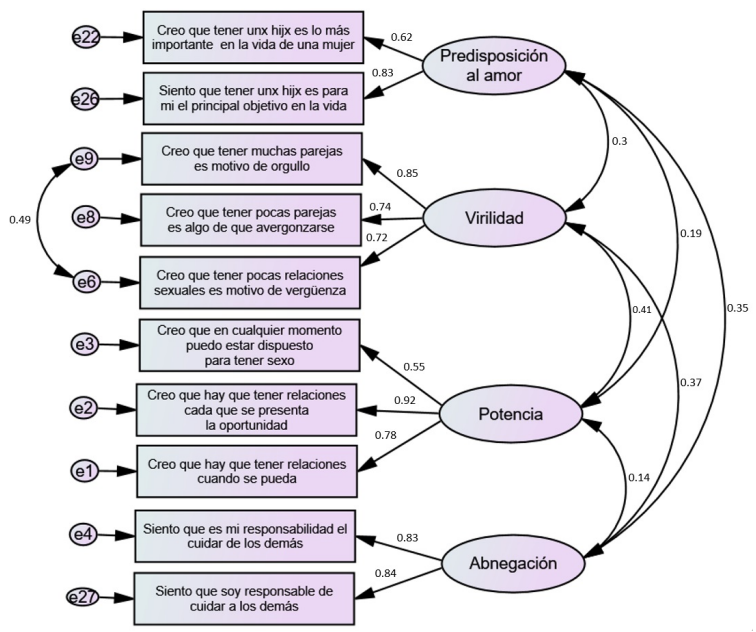

Se aprecia un modelo con elementos e ítems que no cumplen con la relación y carga factorial idónea (Figura 1). Sin embargo, es marcada la relación que se tiene entre dimensiones. Razón por la cual se hicieron distintos modelos previos al definitivo (Figura 2). Eliminando por covarianzas y otras por confiabilidad. Sin embargo, el modelo que se presenta a continuación, se obtuvo mediante el análisis de las covarianzas.

\section{Figura 2}

Modelo factorial confirmatorio definitivo.

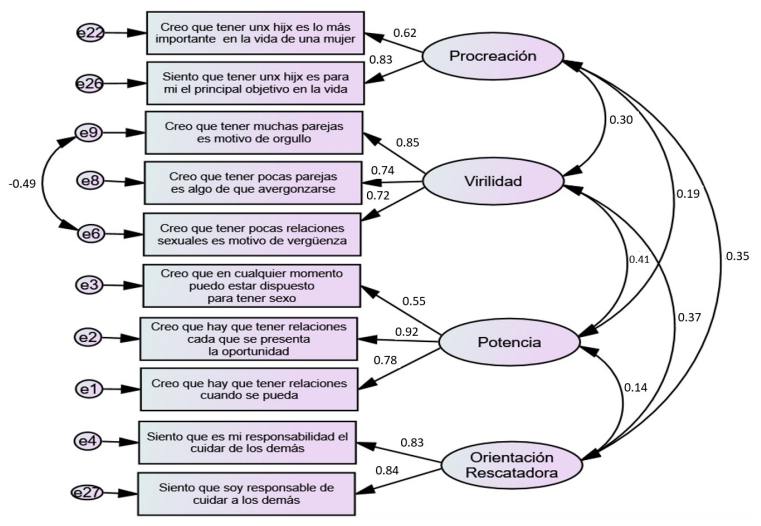

Como se puede observar, posterior a los elementos declarados del modelo factorial, se conservaron aquellos que se mantenían con propiedades psicométricas adecuadas y se cambiaron algunos nombres de factores con base a los ítems que permanecieron en ellos. El modelo final presenta altas cargas factoriales y relaciones consistentes con la teoría y la finalidad del constructo. A continuación, se presentan los descriptivos de cada uno de los ítems del modelo final en la Tabla 4. 
Tabla 4

Descriptivos por ítem de la escala definitiva de mandatos de género

\begin{tabular}{|c|c|c|c|c|c|c|}
\hline Descriptivos & Media & $D E$ & Asimetria & $\begin{array}{r}\text { Error de } \\
\text { asimetria }\end{array}$ & Curtosis & $\begin{array}{l}\text { Error de } \\
\text { curtosis }\end{array}$ \\
\hline $\begin{array}{l}\text { Creo que tener muchas parejas es } \\
\text { motivo de orgullo }\end{array}$ & 1.57 & 0.996 & 1.924 & 0.116 & 3.245 & 0.231 \\
\hline $\begin{array}{l}\text { Creo que tener un hijo(a) es lo más } \\
\text { importante en la vida de una mujer }\end{array}$ & 2.32 & 1.355 & 1.036 & 0.116 & 2.592 & 0.231 \\
\hline $\begin{array}{l}\text { Creo que tener pocas parejas es algo } \\
\text { de que avergonzarse }\end{array}$ & 1.47 & 0.94 & 2.122 & 0.116 & 3.883 & 0.231 \\
\hline $\begin{array}{l}\text { Siento que tener un hijo es para mí el } \\
\text { principal objetivo en la vida }\end{array}$ & 1.92 & 1.184 & 1.067 & 0.116 & 0.022 & 0.231 \\
\hline $\begin{array}{l}\text { Creo que en cualquier momento } \\
\text { puedo estar dispuesto para tener sexo }\end{array}$ & 2.5 & 1.327 & 0.451 & 0.116 & -0.969 & 0.231 \\
\hline $\begin{array}{l}\text { Creo que tener pocas relaciones } \\
\text { sexuales es motivo de vergüenza }\end{array}$ & 1.43 & 0.858 & 2.173 & 0.116 & 4.38 & 0.231 \\
\hline $\begin{array}{l}\text { Creo que hay que tener relaciones } \\
\text { cada que se presenta la oportunidad }\end{array}$ & 2.2 & 1.233 & 0.653 & 0.116 & -0.68 & 0.231 \\
\hline $\begin{array}{l}\text { Creo que hay que tener relaciones } \\
\text { sexuales cuando se pueda }\end{array}$ & 2.43 & 1.272 & 0.458 & 0.116 & -0.855 & 0.231 \\
\hline $\begin{array}{l}\text { Siento que soy responsable de cuidar } \\
\text { a los demás }\end{array}$ & 2.07 & 1.21 & 0.885 & 0.116 & -0.339 & 0.231 \\
\hline $\begin{array}{l}\text { Siento que es mi responsabilidad el } \\
\text { cuidar de los demás }\end{array}$ & 2.07 & 1.154 & 0.894 & 0.116 & -0.08 & 0.231 \\
\hline
\end{tabular}

Como se puede apreciar, los elementos con medias más elevadas corresponden a la actividad sexual y la procreación. Cuestiones íntimamente ligadas pero vividas como un mandato. La posibilidad de evidenciar dichos elementos reitera lo que se propone en Bosh et al. (2013) ser para otros (en la feminidad) que se enlaza a la potencia en las conformaciones de masculinidad: ser para sí mismo.

\section{Conclusión}

Los indicadores de los mandatos de género, permiten desvelar los elementos implícitos en las relaciones sexuales y de pareja. Por tanto, son pautas relacionales que se convertirán en acciones al momento de generar vínculos encontrados en la población de este estudio. El modelo confirmatorio de la escala de mandatos de género permite el acercamiento al impacto que tienen las formas de socialización heteronormativas en las sexualidades y la dimensión relacional de las personas. De igual forma, el instrumento sobre mandatos de género puede ser sumamente útil para detectar el machismo implícito en los determinantes sociales que se encuentran cotidianamente en la sociedad, mismo que impactan en las decisiones de las personas y que quizá incluso puedan encontrarse implicados en la violencia de género, el desempeño sexual, entre múltiples otras formas de relación.
La escala de mandatos de género presenta en general propiedades psicométricas idóneas. Lo cual le permite destacarse entre otras alternativas similares encontradas, como la de López-Cepero et al. (2013) que presenta una menor confiabilidad (global) y no hace referencia al constructo mandatos de género. En contraste con el cuestionario de actitudes hacia la igualdad de género (De Sola \& Meliá, 2003), se encuentran distintos abordajes teóricos y mejores propiedades psicométricas. La escala con finalidad similar es la de asertividad sexual (Torres-Obregon et al., 2017) quienes proponen un instrumento con análisis confirmatorio con buenas propiedades psicométricas, sin embargo, utilizando un constructo muy distinto. Por último, la escala de creencias de género de Latka et al. (2009), elegida por su posible similitud, presenta una baja confiabilidad general y no se conocen sus otras propiedades psicométricas.

El análisis factorial confirmatorio de la escala de mandatos de género dista de su versión exploratoria, sin embargo, mantiene la propuesta original en dimensiones femeninas y masculinas, y la equivalencia entre factores (dos en cada una) que le representan. La escala presenta una propuesta original de utilidad para la intervención clínica y social, y se resaltan los mandatos de género como un continum, por lo cual en los análisis psicométricos se buscó abordar ambos sexos, ya que las conformaciones de masculinidad patriarcales impactan en los mandatos de feminidad y viceversa.

En cuanto a las propiedades psicométricas de la escala, uno de los factores tiene una baja confiabilidad, el resto de las propiedades psicométricas del modelo cumplen con los elementos propuestos Bentler y Bonett (1980) de igual forma que los de Brown (2006) y Byrne (2008) entre otros. Por tanto, el instrumento es válido, confiable y útil para emprender investigaciones como lo que se propone en la tesis de Macías-Valadez y Luna-Lara (2016) reflexiones sobre el género, sexualidad y el Virus de Papiloma Humano. Abordar con instrumentos válidos y confiables la investigación de los mandatos de género, resulta un elemento crucial para poder generar prevención e intervención. 
Como limitantes, el instrumento presenta un factor con baja confiabilidad. Trabajó inicialmente para población universitaria y es necesario conocer si existen nuevos mandatos de género que no estén considerados. El instrumento se encuentra limitado en proporción de población y también no abunda en casos clínicos, así como tampoco presenta una validez de criterio, área en la que se pretende ahondar en próximas investigaciones.

\section{Referencias}

Alfonso, L. (2010). Sexualidad y adolescencia. México: Luis Alfonso Guadarrama Rico. Recuperado de: https://guadarramarico.mx/produccioncientifica/libros/adolescencia-y-sexualidad

Bentler, P. M., \& Bonett, D. G. (1980). Significance test and goodness of fit in the analysis of covariance structures. Psychological Bulletin, 88(3), 588-606. https ://doi.org/10.1037/0033-2909.88.3.588

Bombino, Y. (2013). Estudios sobre sexualidad y género: su visualización en la revista Sexología y Sociedad. Revista sexología y sociedad, 19(1), 1-11. Recuperado de: https://revsexologiaysociedad.sld.cu/in dex.php/sexologiaysociedad/article/view/8/ 54

Bosch, E., Ferrer, V. A., Ferreiro, V., \& Navarro, C. (2013). La violencia contra las mujeres: El amor como coartada. Barcelona: Anthropos.

Briseño, M. L. M. (2011). La construcción de la sexualidad y el género en estudiantes de la escuela Normal bilingüe $e$ intercultural de Oaxaca (ENBIO) (Tesis doctoral). UNAM: México D.F. Recuperado de http://132.248.9.195/ptd20 12/enero/0676160/0676160_A1.pdf

Brown, T. A. (2006). Confirmatory factor analysis for applied research. New York: Guilford Press.

Byrne, B. (2008). Testing for multigroup equivalence of a measuring instrument: A walk through the process. Psicothema, 20(4), 872-882. Recuperado de http://www.psicothema.com/english/psi cothema.asp? $\mathrm{id}=3569$

Carbó, P. A. (2017). Abriendo puertas y ventanas a una perspectiva psicosocial feminista: Análisis sobre la violencia de género. Psicoperspectivas. Individuo y Sociedad, 16(2), 79-90. https://doi.org/10.5027/psicoperspec tivas-Vol16-Issue2-fulltext-1021

De Sola, A., \& Meliá, J. L. (2003). El cuestionario de actitudes hacia la igualdad de géneros (CAIG): elaboración y estudio psicométrico. Anuario de Psicología, 34(1), 101-123. Recuperado de https://www.raco.cat/index.php/Anuari oPsicologia/article/viewFile/61637/88510

García-Cueto, E., Rodríguez-Díaz, F. J., BringasMolleda, C., López-Cepero, J., PaínoQuesada, S., \& Rodríguez-Franco, L. (2015). Development of the Gender Role Attitudes Scale (GRAS) amongst young Spanish people. International journal of clinical and health psychology, 15(1), 61-68. https://doi.org/10.1016/j.ijchp.2014.10.004 Lagarde, M. (1996). Género y feminismo. Desarrollo humano y democracia. España: Horas y horas y el Instituto de la Mujer.

Lagarde, M. (2005). Los cautiverios de las mujeres. Madresposas, monjas, putas, presas y locas. México: UNAM.

Laqueur, T. (1990). La construcción del sexo. Cuerpo y género desde los griegos hasta Freud. Madrid, España: Cátedra.

Latka, M., Meyer-Weitz, A., Fielding, K., Kruger, P., Mogwere, T., \& Churchyard, G. C. (2009). Factors associated with concurrent sexual partnering and condom use are not the same: Results from a representative household survey in Rustenburg, South Africa. Poster, 4th South Africa AIDS Conference. Recuperado de https://www.c-changeprogram.org/ content/gender-scales-compendium/ pdfs/3.\%20Gender\%20Beliefs\%20Scale, $\% 20$ Gender\%20Scales \%20Compendium.pdf

Macías-Valadez, M. G. \& Luna-Lara, M. G. (2016). Reflexiones sobre el género, sexualidad y el Virus de 
Papiloma Humano. Uaricha. Revista de Psicología, 13(31), 56-72. Recuperado de: http://www.revistauaricha.umich.mx/oj s_uaricha/index.php/urp/article/view/9/7

Macías-Valadez, G. M., \& Luna-Lara, M. G. (2018). Validación de una escala de mandatos de género en universitarios de México. Ciencia UAT, 12(2), 67-77. https: //doi.org/10.29059/cienciauat.v12i2.823

Majdalani, M. P., Alemán, M., Fayanás, R., Guedes, A., \& Mejía, R. M. (2005). Validación de un cuestionario breve para detectar situaciones de violencia de género en las consultas clínicas. Revista Panamericana de Salud Pública, 17(2), 79-83. https://doi.org/10.1590/S1020-4989 2005000200003

Martínez-Munguía C. \& Galán, J. S. (2015). Salud, género y violencia: dimensión preventiva. En E., Camacho \& S., Galán. Alternativas psicológicas de intervención en problemas de salud. México: Manual Moderno.

Morokoff, P. J., Quina, K., Harlow, L. L., Whitmire, L., Grimley, D. M., Gibson, P. R., \& Burkholder, G. J. (1997). Sexual Assertiveness Scale (SAS) for women: development and validation. Journal of Personality and Social Psychology, 73(4), 790-804. https://doi.org/10.1037/0022-351 4.73.4.790

López-Cepero, J., Rodríguez-Franco, L., Rodríguez-Díaz, F. J., \& Bringas, C. (2013). Validación de la versión corta del Social Roles Questionnaire (SRQ-R) con una muestra adolescente y juvenil Española. REMA (Revista Electrónica de Metodología Aplicada), 18(1), 1-16.

Rudman, L. A. \& Glick, P. (2008). The social psychology of gender. How power and intimacy shape gender relations. Nueva York: The Guilford Press.

Sanyal, M. M. (2012). Vulva. La revelación del sexo invisible. Madrid: Anagrama

Sierra, J. C., Rojas, A., Ortega, V., \& Martín, O. J. A. (2007). Evaluación de actitudes sexuales machistas en universitarios: primeros datos psicométricos de las versiones españolas de la Double Standard Scale (DDS) y de la Rape Supportive Attitude Scale (RSAS). International Journal of Psychology and Psychological Therapy, 7(1), 41-60.

Torres-Obregon, R., Onofre-Rodríguez, D. J., Sierra, J. C., Benavides-Torres, R. A., \& Garza-Elizondo, M. E. (2017). Validación de la Sexual Assertiveness Scale en mujeres mexicanas. Suma psicológica, 24 (1), 34-41. https://doi.org/10.1016/j.sumpsi.201 7.01 .001

United States Agency for International Development (USAID) \& C-Change Project (2011). Compendium of gender scales. Washington, DC: C-Change.

Yago, T. S. (2011). Jóvenes, anticoncepción y género. Perspectiva de género en la práctica clínica. Zaragoza, ES: Arpirelieve.

Yago, T. S., \& Tomás, C. A. (2013). Condicionantes de género en anticoncepción: diseño y validación de un cuestionario. Atención Primaria 45(8), 418-425. https://doi.org/10.1016/j.aprim.2 013.04 .013

\section{Notas}

* Artículo de investigación. 